\title{
Die laa-Verdoppelung in der Stadt Zug
}

\author{
Mirija Weber (Zürich)
}

\begin{abstract}
The paper deals with the doubling of the verb laa 'let' in the dialect of the city of Zug. The data are collected by means of written questionnaires. The results of this inquiry are compared with the results gained by the project SADS ('Syntactic Atlas of German-speaking Switzerland'). This comparison clearly shows differences in the results according to the elicitation techniques. It also shows that there is a greater variation with regard to the laa-doubling than illustrated by the SADS project. This variation seems to at least correlate with the age of the informants.
\end{abstract}

\section{$1 \quad$ Einleitung}

\subsection{Fragestellung}

Verbverdoppelung ist ein für das Schweizerdeutsche typisches Phänomen, bei dem die Verben ga, cho, laa und aafaa im Zusammenhang mit einem abhängigen Infinitiv wiederholt werden (s. Beispiele 1 und 2). Diese Arbeit behandelt das Phänomen der Verdoppelung des Verbs laa 'lassen' im Dialekt der Stadt Zug. Es existiert bereits eine Datenerhebung zu diesem Phänomen für den Ortspunkt Zug im Rahmen des Projekts "Syntaktischer Atlas der Deutschen Schweiz" (SADS). ${ }^{1}$ In der vorliegender Arbeit wird angestrebt, die Resultate des SADS-Projektes mit denjenigen einer eigenen Untersuchung (MW) für die Stadt Zug zu vergleichen. Untersucht werden in beiden Fällen die Übersetzungsantworten auf die Fragen 1, 3 und 5 des zweiten SADS-Fragebogens (s. Anhang). Dabei steht die Frage im Mittelpunkt, ob die Resultate bei unterschiedlich grossem Stichprobenumfang voneinander abweichen oder nicht. Sind es beim SADS zehn Informanten für den Ortspunkt Zug, so wurden bei der erneuten Untersuchung (MW) 46 Personen befragt. Es geht also um folgende Fragen:

1. Welche Resultate zeigt die Untersuchung des SADS für den Ortspunkt Zug bei den Fragen II.1, II.3 und II.5?

2. Welche Resultate zeigt die MW-Untersuchung diesbezüglich?

3. Sind die Resultate der MW-Untersuchung trotz des grösseren Stichprobenumfangs die gleichen wie beim SADS für die Stadt Zug oder weichen sie davon ab?

4. Falls die Resultate anders ausfallen, worauf ist der Unterschied zurückzuführen?

Nach einem einführenden Abschnitt 1.2, der das Phänomen der Verdoppelung bei laa vorstellt, werden im nachfolgenden Abschnitt 2 die Resultate der SADS-Abfrage wie auch die Methode und die Vorgehensweise der MW-Untersuchung vorgestellt. Im Abschnitt 3 werden die Resultate miteinander verglichen. Verglichen werden die Resultate in toto, die Resultate je Alterskategorie und der Verlauf der Antworten von der ältesten Altersgruppe bis zur jüngsten. Zudem werden die Antworten der einzelnen Informanten zueinander in Beziehung gesetzt,

1 Für eine kurze Beschreibung des SADS siehe www.ds.uzh.ch/dialektsyntax. 
wobei wiederum zwischen SADS und MW-Abfrage verglichen wird. Der Schluss (Abschnitt 4) führt die wichtigsten Befunde aus dem Abschnitt 3 nochmals auf.

\subsection{Die Verdoppelung von laa und ihre Besonderheiten}

Das Phänomen der Verbverdoppelung wird in Lötscher (1993: 180) als Wiederholung der Verben gaa, choo, laa und aafaa in verkürzter Form bezeichnet, wenn diese Verben Infinitive regieren. Dabei treten diese Kurzformen zwischen dem finiten Verb und dem Infinitiv auf [Hervorhebung von MW]:

(1) a. Ich gang der Onkel ga bsueche.

'Ich gehe den Onkel gehen besuchen.'

b. Er chunnt cho der Onkel bsueche.

'Er kommt kommen den Onkel besuchen.'

c. Er laat d Vaase la gheie.

'Er lässt die Vase lassen fallen.'

d. Si faat s Zmittag afa choche.

'Sie fängt das Mittagessen anfangen kochen.'

Schmidt (2000: 1) weist in der Untersuchung zum Zürichdeutschen darauf hin, dass bei der Wiederholung das Verb eine unbetonte und phonetisch reduzierte Infinitivform annimmt (vgl. (1c) la vs. laat bzw. Infinitiv laa). Die laa-Verdoppelung ist funktional nicht gleich geartet wie die von gaa und choo und auch nicht gleich zwingend (cf. Lötscher 1993: 181, 193 und Schmidt 2000: 37); sie wird auch als Variante zur Verwendung ohne Verdoppelung seltener realisiert. Marti (1985: 172) führt für das Berndeutsche aus, dass "[d]ie Reduplikation [...] bei gah/goh zwingend, bei choo mit Ausnahme gewisser Bedeutungsnüancierungen ebenfalls, bei laa/loo üblich [ist]".

Laut Schmidt (2000: 37f.) ist die Verdoppelung von laa sowohl bei Erststellung des flektierten Verbs im Imperativ (2a), als auch bei Spät-/Endstellung der infiniten Form bei Perfektund Modalkonstruktionen (2b) und (2c) möglich:

(2) a. Lans nöd la fale!

b. Er hät d Vaase la gheie laa.

c. Wotsch mi ächt la gaa laa!

Auch der syntaktische Status von laa unterscheidet sich von gaa und choo in bestimmten Situationen. Wenn Fischer (1989: 359) und Weber (1948: 246f.) sagen: "Zum Unterschied von 1 und 2 [ $g a$ und $c h o$ ] bewahren diese Infinitive $\mathrm{z}$. T. ihre ursprüngliche Bedeutung, so in Fällen wie: I chönt s la fale. I wett s na la druf aachoo", so deuten sie damit an, dass eine Modalkonstruktion (oder eine Perfektkonstruktion) wie bei

(3) Er mues de Chopf lo hange.

nicht als elliptische Verdoppelung verstanden werden kann. Denn während bei den Bewegungsverben $g a$ und cho der Infinitiv (oder in Perfektkonstruktionen das Partizip) unterdrückt werden kann, so ist dies bei laa nicht möglich. Bei Sie isch de Bueb cho tröschte ist das Partizip also unterdrückt und cho demnach Verdopplungselement, wohingegen lo in (3) als Infinitiv fungiert. "Nur die 'echten' Verdoppelungen mit zwei Vertretern des Verbs laa können dann als Verdoppelungen gewertet werden" (cf. Schmidt 2000: 41). Eine weitere syntaktische Besonderheit der Verdoppelung von laa ist laut Schmidt (2000: 42) die Tatsache, dass dabei - anders als bei ga und cho - keine zwischen Verdoppelungselement und Infinitiv eingeschobenen Objekte stehen können (vgl. 4a und 4b vs. 4c und 4d). Diese Stellungs- 
variante sei nur dann möglich, wenn die vom Verdoppelungselement abhängige Verbalphrase eine intentional kontrollierbare Handlung bezeichnet.

(4) a. Ich gange es Buech go hole.

b. Ich gange go es Buech hole.

c. *Ich lo lo de Sepp gwünne.

d. Ich lo de Sepp lo gwünne.

Hinsichtlich der Stellung im Satz stellt Schmidt (2000: 65) für das Zürichdeutsche fest, dass im Gegensatz zum Verb ga, cho und aafaa, die bei der Verdoppelung in den meisten Fällen in zentrifugaler (also absteigender) Abfolge auftreten (vgl. gange ... go hole in 4a), das Verb laa eher ein zentripetales (aufsteigendes) Abfolgemuster im Perfekt und in Modalkonstruktionen zeigt (flicken lassen).

Schliesslich hat Schmidt in ihren Untersuchungen festgestellt, dass, je jünger die Sprecher sind, die Tendenz besteht, laa nicht zu verdoppeln. Sie nimmt insofern einen "Sprachwandelprozess in progress" an (cf. Schmidt 2000: 54). Bei denjenigen, die die Verdoppelung noch gebrauchen, bestehe die Tendenz, die zentrifugale (absteigende) Wortfolge aufzugeben. Die Entwicklung scheint über den Verlust des ursprünglichen Musters hin zu einer vollständigen Übernahme der standarddeutschen Variante zu verlaufen (cf. Schmidt 2000: 67).

\subsection{Die räumliche Verteilung}

Die sprachgeographische Situation der Verbverdoppelung allgemein fasst Lötscher wie folgt zusammen:

Sprachgeographisch ist die Verdopplung in den verschiedenen Sprachregionen der Schweiz ungleichmässig durchgeführt. Die Verdopplung mit gaa zeigt die weitaus ausgedehnteste Verbreitung. [...] In der ganzen Schweiz ist nach 'gehen' die Verdopplung mit gaa obligatorisch, in gewissen Rand- und Reliktgebieten auch nach 'kommen'; im mittleren Schweizerdeutsch dagegen ist nach 'kommen' die Verdopplung mit choo das Übliche. [...] Choo und gaa sind ihrerseits wiederum verbreiteter oder zumindest zwingender notwendig als die Wiederholung mit laa [...] Die Verdopplung bei laa scheint in weiten Teilen des Schweizerdeutschen nicht eine voll obligatorische Konstruktion zu sein, sondern als Variante neben Konstruktionen ohne Verdopplung zu existieren. Das Gleiche dürfte wohl für die aafaa-Verdopplung gelten. (Lötscher 1993: 182)

Auskunft über die Verbreitung der laa-Verdoppelung gibt Karte III 263 des SDS. Es galt dabei, den Satz Lass ihn gehen! zu übersetzen. Daraus wird ersichtlich, dass der Innerschweizer Raum eine Kernzone ausmacht, wo Verdoppelung "mit besonderer Häufigkeit" (Lötscher 1993: 196) auftritt (s. Karte 1 im Anhang). Der Südwesten des Aargaus und das Wallis gehören ebenfalls zu dieser Kernzone. Eine zweite Zone, wo "mit relativ grosser Häufigkeit" (Lötscher 1993: 196) verdoppelt wird, umgürtet die Kernzone und erstreckt sich in die Kantone Bern, Solothurn, beide Basel, Aargau und Zürich. Im Westen von Bern und Solothurn kommt die laa-Verdoppelung aber nur vereinzelt vor, während der nördliche Aargau, der grösste Teil des Kantons Zürich, sowie die gesamte Ostschweiz der Zone angehören, die "praktisch ausschliesslich" (Lötscher 1993: 196) die einfache Konstruktion verwendet.

Eine weitere wichtige Quelle ist der Syntaktische Atlas der Deutschen Schweiz (SADS). Darin behandeln drei Fragen das Phänomen der laa-Verdoppelung. Die Übersetzung von $I h r$ dürft alles liegen lassen mit einer Imperativkonstruktion zeigt weitgehend das gleiche Bild wie SDS (III 263). Mit Lönd alles lo ligge wird im Westen, Nordwesten, im Aargau und in der Zentralschweiz, vereinzelt in Zürich übersetzt. Lönd alles ligge ist hingegen in der gesamten Ostschweiz, im grössten Teil von Zürich und im nördlichen Grenzgebiet des 
Aargau und beider Basel, ganz vereinzelt auch im Westen und in der Zentralschweiz auszumachen (s. Karte 2 im Anhang 2). Die übrigen Abfragen zeigen Folgendes:

- Die verdoppelte Übersetzung der Indikativkonstruktion im Präsens Er lässt den Schreiner kommen erscheint dicht - allgemein und je Ortspunkt - im Westen, Nordwesten, im Aargau, in der Zentralschweiz und am westlichen Rand des Kantons Zürich, vereinzelt und dicht je Ortspunkt auch in Graubünden. Keine Verdoppelung findet man in der Ostschweiz, im grössten Teil von Zürich, am nordöstlichen Rand der Zentralschweiz, im nördlichen Aargau und in beiden Basel. Keine Verdoppelung erscheint vereinzelt und weniger dicht je Ortspunkt auch im Aargau und im Westen.

- Sowohl Perfekt- wie Modalkonstruktion führen dann zu einem drastischen Rückgang der Verdoppelung. Hast du die Uhr flicken lassen und Ihr dürft alles liegen lassen werden nur sehr vereinzelt und wenig dicht je Ortspunkt im Aargau, in der Zentralschweiz, am östlichen Rand von Zürich und in Graubünden verdoppelt übersetzt.

- Eine weitere Aussage ermöglicht der SADS zur Wortstellung. Es zeigt sich, dass die räumliche Verteilung der absteigenden Verbalfolge am rechten Satzrand in Hesch d Uhr lo flicke und Ir döfed alles lo ligge mit der Verteilung des verdoppelten Er loot de Schriner lo cho korrespondiert. Die aufsteigende Verbalfolge (Hesch d Uhr flicke loo, Ir döfed alles ligge loo) deckt sich mit dem einfachen, nicht verdoppelten Er loot de Schriner cho. Diese Korrespondenz gilt aber für den Aargau und den Westen nicht (cf. Glaser/Frey 2007: 6f.).

Zusammenfassend kann gesagt werden, dass sich die vom SDS präsentierten und von Lötscher diskutierten Kernzonen der Verdoppelung im Zentrum und im Westen mit der OstWest-Verteilung des SADS (einfache Konstruktion und aufsteigende Wortfolge im Osten, Verdoppelung und absteigende Wortfolge im Westen) weitgehend decken. Die einfache Übersetzung Er loot de Schriner cho im Aargau und im Westen könnte schliesslich parallel zu Lötschers Beobachtung der "abschwächende[n] Häufigkeit der 'lassen'-Verdopplung westlich des mittleren Streifens, insbesondere auch am Westrand des Kantons Bern" (Lötscher 1993: 196) gesehen werden.

\section{Methoden und Vorgehensweise}

\subsection{Fragebogen}

Die Datenerhebung für die vorliegende Arbeit basiert auf dem zweiten Fragebogen des SADS-Projekts. Drei Übersetzungsaufgaben (II.1, II.3 und II.5 hier als (5a), (5b) und (5c) wiedergegeben) thematisieren darin die laa-Verbverdoppelung: ${ }^{2}$

(5) a. Hast du die Uhr flicken lassen? (II.1)

b. Er lässt den Schreiner kommen. (II.3)

c. Ihr dürft alles liegen lassen. (II.5)

Diese Übersetzungsaufgaben wurden für die erneute Untersuchung unverändert übernommen, um die Resultate besser vergleichen zu können. Ebenso unverändert übernommen wurden die Fragen zu den persönlichen Angaben, die primär überadäquate oder nicht adäquate Informanten entscheiden (s. den Vorspann zum Fragebogen im Anhang). Der Fragebogen wurde den Informanten per E-Mail zugestellt und von diesen - schriftlich bearbeitet - auf die gleiche Art retourniert.

\footnotetext{
2 S. Fragebogen im Anhang 1.
} 


\subsection{Informanten}

Es wurden rund 150 Personen im Kanton Zug angeschrieben. ${ }^{3}$ Von diesen 150 Personen wurden für den Vergleich 46 Personen ausgesucht. ${ }^{4}$ Dabei waren die ausschlaggebenden Kriterien der Ort des Aufwachsens und die Dialektselbsteinschätzung.

Da die vorliegende Arbeit den Ortspunkt Stadt Zug untersucht, mussten die Informanten in der Stadt Zug aufgewachsen sein. Zudem wurden nur Personen berücksichtigt, die ihren Dialekt als "Zugerdeutsch" bezeichneten. ${ }^{5}$ Um die Kriterien der eigenen Untersuchung weiter an den Standard derjenigen des SADS-Projektes anzugleichen, wurde auch die demologische Zusammensetzung der Stadtzuger SADS-Informantengruppe übernommen. Segmentiert in drei Alterskategorien sieht die Zusammensetzung wie folgt aus:

\begin{tabular}{|l|l|r|r|}
\hline Kategorie & Alter & SADS Pers. (in \%) & MW Pers. (in\%) \\
\hline A & $20-39$ & $3(30)$ & $13(28)$ \\
B & $40-59$ & $2(20)$ & $12(26)$ \\
C & $>60$ & $5(50)$ & $21(46)$ \\
\hline Total & & $10(100)$ & $46(100)$ \\
\hline
\end{tabular}

Tab. 1: Alterskategorien der SADS- und der MW-Untersuchung

Weitere Kriterien, wie der Dialekt der Eltern, der momentane Wohnort oder längere Abwesenheiten, wurden wie im SADS-Projekt ebenfalls abgefragt.

\subsection{Schwierigkeiten}

Die wohl grösste Schwierigkeit bei der Datenerhebung war das Finden von passenden Informanten. Obwohl 150 Personen angeschrieben wurden, konnte nur rund ein Drittel davon als Informanten herangezogen werden. Ein weiteres Problem in diesem Zusammenhang war der Datenschutz. Sowohl die Pro Senectute Kanton Zug als auch die Korporation Zug durften auf die Anfrage keine Adress- oder Verteilerlisten weitergeben. Ausserdem konnten viele Informanten ihren Dialekt nicht eindeutig bestimmen. Keine oder bloss sehr vage Angaben waren das Resultat. In solchen Fällen wurden die Antworten dieser Personen für den Vergleich nicht berücksichtigt.

\section{$3 \quad$ Resultate, Vergleich und Diskussion}

\subsection{Frage II.1: Hast du die Uhr flicken lassen?}

Verglichen werden die Antworten der 10 SADS-Informanten und der 46 MWInformanten zu der Übersetzungsfrage II.1 Hast du die Uhr flicken lassen? (5a). Tabelle 2 zeigt die Resultate zu dieser Aufgabe in Bezug auf die Verdoppelung von laa und auf die Verbabfolge, wenn laa nicht verdoppelt wird.

3 Es wurden dabei drei Serviceclubs, Lehrkräfte, Familie, Freunde und Bekannte angeschrieben.

4 Es war geplant, mindestens 40 Informanten zu befragen.

5 Es ist klar, dass die durch die Informanten vorgenommene Dialektbezeichnung nicht vor 'falschen' Bezeichnungen feit. Bei der Datenerhebung für diese Arbeit ging es aber in erster Linie darum, klar nicht-zugerische Dialekte auszuschliessen. 


\begin{tabular}{|c|c|c|c|c|c|c|c|c|c|}
\hline \multirow[t]{3}{*}{ Kat. } & \multirow[t]{3}{*}{ Alter } & \multicolumn{4}{|c|}{ SADS-Abfrage } & \multicolumn{4}{|c|}{ MW-Abfrage } \\
\hline & & \multirow[t]{2}{*}{ Verd. } & \multicolumn{3}{|c|}{ Verbabfolge } & \multirow[t]{2}{*}{ Verd. } & \multicolumn{3}{|c|}{ Verbabfolge } \\
\hline & & & $\% \downarrow$ & $\% \uparrow$ & total & & $\% \downarrow$ & $\% \uparrow$ & total \\
\hline $\mathbf{A}$ & $20-39$ & keine & 100 & 0 & 1 & $2 / 12$ & 20 & 80 & 10 \\
\hline B & $40-59$ & keine & 100 & 0 & 2 & $3 / 10$ & 43 & 57 & 7 \\
\hline $\mathbf{C}$ & $>60$ & keine & 100 & 0 & 4 & keine & 78 & 22 & 18 \\
\hline \multicolumn{2}{|l|}{ Total } & keine & 100 & 0 & 7 & $5 / 40$ & 54 & 46 & 35 \\
\hline
\end{tabular}

Tab. 2: Resultate zu Übersetzungsaufgabe II.1 der SADS- und der MW-Abfrage; $\downarrow=$ absteigend: la flicke, $\uparrow=$ aufsteigend: flicke laa

Die Resultate lassen sich wie folgt beschreiben: laa wird von den Informanten im Alter zwischen 20 und 39 weder bei der SADS- noch bei der MW-Abfrage verdoppelt. Alle drei SADS-Informanten geben dabei (bei Nicht-Verdoppelung) die absteigende Verbabfolge (100\% !) an. Bei der MW-Abfrage geben 31\% der Informanten (4 von 13) die absteigende und 69\% (9 von 13) die aufsteigende Verbabfolge an. In der Kategorie B der SADS-Abfrage gibt ein Informant (von zwei) die absteigende und einer die aufsteigende Verbabfolge an. In beiden Fällen wird laa nicht verdoppelt. Bei derMW-Abfage umfasst die Kategorie B 12 Personen, von denen eine das Verb laa verdoppelt. Von den restlichen 11 geben 7 Informanten (64\%) die absteigende und vier (36\%) die aufsteigende Verbabfolge an. In der Kategorie C weisen die Antworten der SADS-Abfrage weder Verdoppelung noch die aufsteigende Verbabfolge auf. In der MW-Abfrage dagegen ist das Verhältnis bei der Verbabfolge 18 verbal absteigend (86\%) zu 3 verbal aufsteigend (14\%) bei insgesamt 21 Informanten.

Hinsichtlich der Verdoppelung zeigt die MW-Abfrage im Prinzip die gleiche Tendenz wie bei der SADS-Abfrage, das Verb laa nicht zu verdoppeln (45 von 46). Hinsichtlich der Verbabfolge kann man bei den Informanten der MW-Abfrage die Tendenz beobachten, die aufsteigende Reihenfolge zu gebrauchen (36\%, 16 von 45), während diese Abfolge in der SADSAbfrage nur von einem der zehn Informanten angegeben wird. Ausserdem fällt auf, dass diese Verbabfolge vor allem von der Kategorie A, also jüngeren Informanten, häufiger verwendet wird (69\% vs. $36 \%$ in B und 14\% in C). Der Grund für diese 'Abweichung' könnte der Einfluss des elterlichen Dialekts sein. $78 \%$ derjenigen, die die aufsteigende Verbalfolge verwenden, weisen ihren Eltern Dialekte aus der Ostschweiz und aus Zürich zu (s. Abschnitt 1.3). In zwei Fällen spricht die Mutter Hochdeutsch. ${ }^{6}$ In $43 \%$ dieser Fälle sind es gar beide Elternteile, die nicht Zugerdeutsch sprechen. Im Gegensatz dazu lassen sich bei den SADSInformanten keine derartigen Einflüsse feststellen. Nur in einem Fall wird der Dialekt väterlicherseits als Sanktgallerisch bezeichnet. Diese Neigung der jüngeren Informanten, die aufsteigende Verbabfolge vermehrt zu verwenden, bestätigt die gleiche Beobachtung in Schmidt (2000).

\subsection{Frage II.3: Er lässt den Schreiner kommen}

Bei dieser Frage lässt sich der Vergleich ausschliesslich zur Verdoppelung des Verb laa anstellen. Dabei geht es um folgende Option: Er loot de Schriiner lo cho.

6 Die Standardsprache kennt keine Verbverdoppelung, auch ist die aufsteigende Verbalfolge für sie typisch. 


\begin{tabular}{|l|c|r|r|r|r|r|r|}
\hline \multirow{2}{*}{ Kat. } & \multirow{2}{*}{ Alter } & \multicolumn{3}{|c|}{ SADS-Abfrage } & \multicolumn{3}{|c|}{ MW-Abfrage } \\
\cline { 3 - 8 } & & Verd. \% & \multicolumn{1}{|c|}{ GP } & total GP & Verd. \% & GP & total GP \\
\hline A & $20-39$ & 100 & 3 & 3 & 31 & 4 & 13 \\
B & $40-59$ & 5 & 1 & 2 & 58 & 7 & 12 \\
C & $>60$ & 80 & 4 & 5 & 74 & 14 & 19 \\
\hline Total & & 80 & 8 & 10 & 57 & 25 & 44 \\
\hline
\end{tabular}

Tab. 3: Verdoppelung von laa bei der Übersetzungsaufgabe II.3

Insgesamt zeigt der Vergleich, dass die Gewährspersonen (GP) der SADS-Abfrage häufiger (80\%) verdoppeln als die Informanten bei der MW-Abfrage (57\%). Am besten ist dieser Unterschied bei der Alterskategorie A zu sehen: 100\% (SADS) vs. 31\% (MW). Auch hier könnte der Grund für die Nicht-Verdoppelung (69\%) bei den elterlichen Dialektverhältnissen liegen: Wieder beschreiben $78 \%$ derjenigen, die das Verb laa nicht verdoppeln, den Dialekt ihrer Eltern als Ostschweizerisch, Zürichdeutsch (s. Abschnitt 1.3). In zwei Fällen spricht die Mutter Hochdeutsch. In den Kategorien B und C liegt das Verhältnis der beiden Abfragen relativ nah: $50 \%$ beim SADS zu $58 \%$ bei der MW-Abfrage bzw. $80 \%$ zu $74 \%$. In der Kategorie C wurden die Antworten zweier Informanten der MW-Abfrage nicht berücksichtigt (19 statt 21), weil sie eine Perfektkonstruktion verwendet haben: Er het de Schriiner lo choo und Er het de Schriiner choo loo.

Bei der Frage II.3 zeigt sich wiederum die Tendenz der jüngeren (Kat. A) gegenüber den älteren Sprechern (Kat. B und C), das Verb laa nicht zu verdoppeln.

\subsection{Frage II.5: Ihr dürft alles liegen lassen}

Bei dieser Frage hat ein Teil der Informanten den Satz im Imperativ übersetzt: Lönd alles lo ligge! bzw. Lönd alles ligge! Dies wird beim Vergleich berïcksichtigt. Tabelle 4 fasst die Resultate für die Verdoppelung (Verd.) zusammen, wenn kein Imperativ verwendet wurde, und die Resultate zur Verbabfolge für den Fall, dass keine Verdoppelung und kein Imperativ verwendet wurden.

Die SADS-Resultate zeigen bei Frage II.5 insgesamt keine Verdoppelung im Indikativ. Bei der MW-Abfrage wird die Verdoppelung von fünf aus 40 Gewährspersonen $(=12,5 \%)$ verwendet. Ganz anders ist das Bild bei der Verbabfolge: Die SADS-Informanten wählen ausschliesslich die absteigende Folge, bei der MWAbfrage geben 54\% der 35 Informanten die absteigende und $46 \%$ die aufsteigende Verbabfolge an.

\begin{tabular}{|c|c|c|c|c|c|c|c|c|c|}
\hline \multirow[t]{3}{*}{ Kat. } & \multirow[t]{3}{*}{ Alter } & \multicolumn{4}{|c|}{ SADS-Abfrage } & \multicolumn{4}{|c|}{ MW-Abfrage } \\
\hline & & \multirow[t]{2}{*}{ Verd. } & \multicolumn{3}{|c|}{ Verbabfolge } & \multirow[t]{2}{*}{ Verd. } & \multicolumn{3}{|c|}{ Verbabfolge } \\
\hline & & & $\% \downarrow$ & $\% \uparrow$ & total & & $\% \downarrow$ & $\% \uparrow$ & total \\
\hline $\mathbf{A}$ & $20-39$ & keine & 100 & 0 & 1 & $2 / 12$ & 20 & 80 & 10 \\
\hline B & $40-59$ & keine & 100 & 0 & 2 & $3 / 10$ & 43 & 57 & 7 \\
\hline C & $>60$ & keine & 100 & 0 & 4 & keine & 78 & 22 & 18 \\
\hline \multicolumn{2}{|l|}{ Total } & keine & 100 & 0 & 7 & $5 / 40$ & 54 & 46 & 35 \\
\hline
\end{tabular}

Tab. 4: Resultate zu Übersetzungsaufgabe II.5 der SADS- und der MW-Abfrage; $\downarrow=$ absteigend: la ligge, $\uparrow=$ aufsteigend: ligge laa

In der Alterskategorie A kommt beim SADS keine Verdoppelung im Indikativ vor. Bei der MW-Abfrage kommt die Verdoppelung in zwei von 12 Fällen vor $(17 \%)$, der Rest der GP (83\%) verdoppelt nicht. Die Verbalfolge ist beim SADS zu $100 \%$ absteigend. In der MW- 
Untersuchung ist sie dies nur zu 20\%. 80\% bevorzugen die aufsteigende Verbalfolge. In $75 \%$ dieser Fälle wird den Eltern ein Ostschweizer oder Zürcher Dialekt zugewiesen, zwei Mütter sprechen Hochdeutsch.

In der Alterskategorie B des SADS ist keine Verdoppelung im Indikativ registriert, während bei der MW-Abfrage die Verdoppelung von drei GP aus $10(\approx 33 \%)$ angegeben wird. Diesmal kann der Grund dafür aber nur schwerlich die elterlichen Dialektverhältnisse derjenigen, die verdoppeln, betreffen: Die Verhältnisse sind nämlich bei den SADS-Informanten wie bei den MW-Informanten die gleichen, mit Ausnahme von zwei MW-Informanten, die einen Sanktgallerdeutsch sprechenden Vater ausweisen. Absteigend übersetzen beim SADS 100\%, bei den MW-Informanten nur 43\% der GP. Auch hier kann der elterliche Dialekt nicht der Grund sein, die MW-Informanten weisen die gleichen Verhältnisse auf wie die SADS-Informanten.

Bei der Kategorie $\mathrm{C}$ zeigen sowohl die SADS-Daten als auch die in der MW-Untersuchung vorliegenden Resultate keine Verdoppelung im Indikativ. Absteigend wird beim SADS zu $100 \%$ übersetzt, während bei MW-Abfrage es zu 78\% der Fall ist. 22\% der MW-Informanten dieser Altersgruppe verwenden die aufsteigende Verbalfolge. Die Hälfte davon hat Eltern, die einen Ostschweizer oder Zürcher Dialekt sprechen. Eine der vier Personen lebte während eines Jahres im Thurgau. Die Ostschweizer Einflüsse könnten zur Abweichung geführt haben, wenn man bedenkt, dass die SADS-Informanten im Gegensatz zu MW alle ortsansässig sind.

Bei der Verdoppelung im Indikativ ist beim SADS von $\mathrm{C}$ nach A keine Tendenz auszumachen, es wird nirgends verdoppelt. Auch in der MW-Untersuchung kann man keine Tendenz feststellen. In den Kategorien A und B wird laa im Gegensatz zu C jedoch verdoppelt. Hinsichtlich der Verbalfolge erkennt man wiederum keine Tendenz beim SADS, während sich bei der MW-Untersuchung eine klare Tendenz abzeichnet: Von C nach A kommt die absteigende Verbalfolge deutlich seltener vor. Verantwortlich dafür könnte der Einfluss der Dialekte der Eltern bei einem Teil der GP in den Kategorien A und B sein.

Bei der Übersetzung mit Imperativ (Lönd alles lo ligge und Lönd alles ligge) verwenden beim SADS 75\% der GP die Verdoppelung (s. Tab. 5). Bei der MWUntersuchung liegt dieser Wert bei $67 \% .^{7}$

\begin{tabular}{|l|l|l|l|l|l|l|l|}
\hline \multirow{2}{*}{ Kat. } & \multirow{2}{*}{ Alter } & \multicolumn{3}{c|}{ SADS-Abfrage } & \multicolumn{3}{c|}{ MW-Abfrage } \\
\cline { 3 - 8 } & & Verd. \% & $=$ GP & total GP & Verd. \% & = GP & total GP \\
\hline A & $20-39$ & 100 & 2 & 2 & 100 & 1 & 1 \\
B & $40-59$ & 100 & 1 & 1 & 50 & 1 & 2 \\
C & $>60$ & 0 & 0 & 1 & 67 & 2 & 3 \\
\hline Total & 75 & 3 & 4 & 67 & 4 & 6 \\
\hline
\end{tabular}

Tab. 5: Verdoppelung von laa bei der Übersetzungsaufgabe II.5 (Imperativ)

In der Alterskategorie A wird sowohl bei der SADS-Abfrage wie auch bei der MW-Untersuchung im Imperativ das Verb laa zu 100\% verdoppelt. In der Kategorie B liegt beim SADS ein Fall der Übersetzung mit Imperativ mit Verdoppelung vor, bei der MW-Abfrage übersetzen zwei GP mit Imperativ. Dabei wird die Verdoppelung von einem Informanten verwendet. Die Mutter dieses Informanten ist Sanktgallerin. In der Kategorie C das SADS übersetzt ein Informant mit Imperativ ohne Verdoppelung. Bei der MW-Abfrage verdoppeln zwei GP von drei (Kat. C) das Verb laa im Imperativ. Die Dialektsituation der Eltern verhilft hier jedoch zu keiner schlüssigen Erklärung.

7 Die Prozentangaben sind mit Vorsicht zu geniessen, da die absoluten Zahlen sehr gering sind. 
Beim SADS wird im Imperativ bei A und B zu je 100\% verdoppelt, bei C überhaupt nicht. Bei den MW-Resultaten zeigt sich ein anderer Verlauf, wenn sich von $\mathrm{C}$ nach A die Verdoppelung im Imperativ von $67 \%$ über $50 \%$ bis zu $100 \%$ verändert.

\subsection{Vergleich der Antworten}

Im Folgenden werden die Antworten der einzelnen Informanten zueinander in Beziehung gesetzt: Die Informanten der Alterskategorie A des SADS verwenden bei Frage II.1 in toto die absteigende Verbalfolge (s. Tab. 2), verdoppeln bei Frage II.3 (s. Tab. 3) und verwenden bei Frage II.5 wiederum die absteigende Verbalfolge ohne Verdoppelung, falls sie den Indikativ wählen (s. Tab. 4). Wird mit Imperativ übersetzt, so wird in allen Fällen die Verdoppelung verwendet (s. Tab. 5). Bei den MW-Informanten der Kategorie A zeigt sich das gleiche Bild: Wer bei Frage II.1 absteigend übersetzt (s. Tab. 2), verdoppelt bei Frage II.3 (s. Tab. 3) und wählt bei Frage II.5 die absteigende Verbalfolge oder aber verdoppelt im Indikativ: Er loot de Schriiner lo cho (s. Tab. 4). Dementsprechend gilt hier: Wer bei II.1 aufsteigend übersetzt, verdoppelt bei II.3 nicht und übersetzt auch bei II.5 aufsteigend. Dieses Muster (Verdoppelung/absteigend; keine Verdoppelung/aufsteigend) ist das gleiche wie das gesamtschweizerisch dominierende (s. Abschnitt 1.3 und Karten im Appednix 2).

Gleiches zeigt sich bei der Kategorie B des SADS: Die Gewährsperson, die Hesch d Uhr lo flicke sagt, sagt auch Er loot de Schriiner lo cho und Ihr döfed alles lo ligge. Zusätzlich nennt dieser Informant auch Lönd alles lo ligge als mögliche Übersetzung bei Frage II.5. Bei der zweiten Gewährsperson sind die Antworten aber nicht gleich konsequent: Der Informant wählt die aufsteigende Verbalfolge bei II.1 (flicke laa), verdoppelt bei II.3 nicht (Er loot de Schriiner cho), wählt dann aber bei II.5 die absteigende Verbalfolge (la ligge). ${ }^{8}$ Die MWInformanten der Kategorie B bestätigen dann einmal mehr das Muster: Wer absteigend übersetzt z. B. la ligge (wenn er es denn macht: Eine Informantin verdoppelt durchwegs - bei II.1, II.3 und II.5 im Indikativ), verdoppelt bei II.3 (vgl. 43\% $\downarrow=3$ GP von 7 und Verdoppelung bei 3 von 10 GP in Tab. 4). Wer aufsteigend übersetzt, verdoppelt nicht.

Bei der Kategorie C des SADS zeigt sich das gleiche Bild. Eine Diskrepanz gibt es aber auch hier: Der Informant, der bei II.3 nicht verdoppelt und bei II.5 einen nicht verdoppelten Imperativ wählt, verwendet bei II.1 die absteigende Verbalfolge (la flicke). ${ }^{9}$ DieMW-Untersuchung zeigt wiederum deutlich im Grossen und Ganzen das oben beschriebene Muster: Wer absteigend übersetzt, verdoppelt, und wer aufsteigend übersetzt, verdoppelt das Verb laa nicht.

\section{$4 \quad$ Zusammenfassung}

Es sollen im Folgenden nochmals tabellarisch (s. Tabelle 6) die wichtigsten Unterschiede zwischen den SADS-Resultaten und denjenigen der MW-Untersuchung aufgeführt werden: ${ }^{10}$ Gemeinsam ist den SADS- und den MW-Resultaten das Muster, das sich ergibt, setzt man die Antworten zueinander in Beziehung: Wer laa verdoppelt, verwendet die absteigende Verbalfolge, wer nicht verdoppelt, verwendet die aufsteigende Verbalfolge (oder je umgekehrt). Dieses Muster ist sowohl beim SADS als auch bei der MW-Untersuchung kategorienübergreifend und fast ausnahmslos auszumachen. Bei den Alterskategorien B und C des SADS gibt es jedoch je einen diskrepanten Antwortenverlauf: Beide Informanten verwenden keine Verdoppelung bei Frage II.3, übersetzen das eine Mal dem Muster entsprechend aufsteigend,

8 Der Grund ist nicht klar: Der Informant ist in Zug aufgewachsen, seine Eltern ebenfalls, auch wohnt er in Zug.

9 Auch hier kann das Verhalten weder auf Wohnort noch auf den Dialekt der Eltern zurückgeführt werden.

10 Nicht aufgeführt werden hier die Unterschiede in toto, da sie von den Unterschieden in den Alterskategorien abhängen, zudem werden auch zwei uninteressante Unterschiede nicht aufgeführt. 
das andere Mal aber absteigend. D.h. gelegentliche absteigende Wortfolge bedingt noch keine Verdoppelung.

\begin{tabular}{|l|l|l|}
\hline \multicolumn{1}{|c|}{ Frage/Phänomen } & \multicolumn{1}{c|}{ SADS } & \multicolumn{1}{c|}{ MW-Abfrage } \\
\hline II.1/Verbalfolge $\downarrow$ & A $100 \%$ & A 31\% \\
II.1/Verbalfolge & C $\rightarrow$ A keine Tendenz & C $\rightarrow$ A Tendenz von $\downarrow$ zu $\uparrow$ \\
\hline II.3/Verdoppelung & A 100\% & A 31\% \\
II.3/Verdoppelung & C $\rightarrow$ A keine Tendenz & C $\rightarrow$ A Tendenz von Verd. zu Nichtverd. \\
\hline II.5/Verbalfolge $\downarrow$ & A $100 \%$ & A 20\% \\
II.5/keine Verd. im Ind. & B 100\% & B 70\% \\
II.5/Verbalfolge $\downarrow$ & B $100 \%$ & B 43\% \\
II.5/Verbalfolge $\downarrow$ & C 100\% & C 78\% \\
II.5/keine Verd. im Imp. & C $100 \%$ & C 33\% \\
II.5/Verbalfolge & C $\rightarrow$ A keine Tendenz & C $\rightarrow$ A Tendenz von $\downarrow$ zu $\uparrow$ \\
\hline
\end{tabular}

Tab. 6: Zusammenfassung der Resultate der SADS- und der MW-Abfrage

Um die zentrale Frage der vorliegenden Arbeit zu beantworten, ob nämlich die Resultate der MW-Untersuchung mit denjenigen der SADS-Abfrage übereinstimmen oder nicht, muss ein Blick auf die Anzahl der gemachten Vergleiche geworfen werden. Insgesamt wurden 32 Vergleiche gemacht. Dabei sind die Resultate in 17 Fällen ähnlich oder die gleichen, in 15 weichen sie klar voneinander ab. Mitgezählt sind dabei auch die Vergleiche in toto; ohne diese gesamthaften Vergleiche, die ja von den Verhältnissen der Alterskategorien abhängig sind, sind es 26 Vergleiche, wovon in 14 Fällen die Resultate ähnlich oder gleich sind und in zehn Fällen klare Unterschiede festgestellt werden können (s. Tab. 6). Es ist eine deutliche Diskrepanz zwischen dem Gesamtresultat des SADS und demjenigen der eigenen Untersuchung zu sehen, wonach die eingangs gestellte Frage, ob die Resultate der beiden Untersuchungen übereinstimmen, mit nein beantwortet werden muss.

Den wichtigsten Unterschied machen die Resultate der Alterskategorie A aus (s. Tab. 6). Es zeigt sich, dass sechs von zehn Mal die Alterskategorie A für die Unterschiede verantwortlich ist. Dies ist auch der Fall bei den Tendenzunterschieden. Die Kategorie A ist also deutlich öfter für diskrepante Resultate verantwortlich als B und C und somit der Hauptgrund für die Diskrepanz des Gesamtresultats (15 Unterschiede bei 32 Vergleichen resp. 10 Unterschiede bei 26 Vergleichen). Es handelt sich dabei um die Phänomene der Verbalfolge im Indikativ Perfekt (II.1 Hast du die Uhr flicken lassen?) und im Indikativ mit Modalverb (II.5 Ihr dürft alles liegen lassen) und um das Phänomen der Verdoppelung im Indikativ Präsens (II.3 Er lässt den Schreiner kommen).

Die Gründe für die Unterschiede bei A dürften beim Dialekt der Eltern zu suchen sein.11 Jeweils rund Dreiviertel der A-Informanten weisen ihren Eltern Ostschweizer oder Zürcher Dialekte, zweimal die Standardsprache zu. In 43\% dieser Fälle sind es gar beide Elternteile, die nicht Zugerdeutsch sprechen. Im Gegensatz dazu lassen sich bei den A-Informanten des SADS keineswegs Einflüsse dieser Art feststellen, da hier als Voraussetzung Ortsfestigkeit möglichst in zweiter Generation bestand.

Um die Frage zu klären, ob die Unterschiede bei der Kategorie A tatsächlich auf die Informantenauswahl oder allenfalls doch auf den vergrösserten Stichprobenumfang zurückzuführen sind, bedürfte es einer weiteren Untersuchung. Dabei müssten die Parameter genau die

11 Welche Rolle der Dialekt der Eltern beim Syntaxerwerb spielt, ist aufgrund mangelnder Untersuchungen (vgl. de Vogelaer im Druck) zum dialektalen Spracherwerb noch nicht zu beantworten. 
gleichen sein wie die der A-Informanten des SADS; den Eltern dieser Informanten dürfte also kein anderer Dialekt als Zugerdeutsch zugewiesen werden.

\section{Literatur}

Fischer, Ludwig (1989): Luzerndeutsche Grammatik. Ein Wegweiser zur guten Mundart. Hitzkirch: Comenius.

Glaser, Elvira/Frey, Natascha (2007): "Doubling Phenomena in Swiss German Dialects". Papers of Workshop Syntactic Doubling in European Dialects, March 16-18, 2006. Amsterdam. www.meertens.knaw.nl/projecten/edisyn/Online_proceedings/Paper_Glaser-Frey.pdf, Stand April 2011.

Id. (1881ff.): Schweizerisches Idiotikon. Wörterbuch der schweizerdeutschen Sprache. Frauenfeld.

Lötscher, Andreas (1993): "Zur Genese der Verbverdopplung bei gaa, choo, laa, aafaa im Schweizerdeutschen". In: Abraham, Werner/Bayer, Josef (eds.): Dialektsyntax. Opladen: 180-200.

Marti, Werner (1985): Berndeutsch-Grammatik für die heutige Mundart zwischen Thun und Jura. Bern.

Schmidt, Christa (2000): Die Verbverdoppelung im Zürichdeutschen. Magisterarbeit, Universität Freiburg i. Br.

SDS = Sprachatlas der deutschen Schweiz. Hg. von Rudolf Hotzenköcherle. Bd. III. Bern: 1975.

Vogelaer, Gunther de (im Druck): "A dialect continuum in child language". In: Glaser, Elvira/ Schmidt, Jürgen E./Frey, Natascha (eds.): Dynamik des Dialekts - Wandel und Variation. Akten des 3. Kongresses der Internationalen Gesellschaft für Dialektologie des Deutschen (IGDD). Stuttgart:.

Weber, Albert (1948): Zürichdeutsche Grammatik. Ein Wegweiser zur guten Mundart. Unter Mitwirkung von Eugen Dieth. Zürich.

\section{Anhang 1: Fragebogen MW}

\section{Vorspann}

Name:

Vorname:

Strasse:

PLZ, Ort:

Tel. (für Rückfragen):

E-Mail-Adresse (für Rückfragen):

Geburtsjahr:

Gelernter Beruf:

Ausgeübter Beruf:

Falls Sie zur Arbeit pendeln: Wohin?

Sie sind aufgewachsen in

Sie sprechen den Dialekt von

Ihre Mutter spricht den Dialekt von

Ihr Vater spricht den Dialekt von

Lebten Sie längere Zeit in einem anderen Dialektgebiet? Wo?

Von wann bis wann? 


\section{Übersetzungsaufgaben (aus SADS II.1, II.3 und II.5)}

1. Sie sind bei Ihrer Tante zu Besuch. In der Stube hängt eine antike Wanduhr, die früher immer kaputt war. Heute aber läuft sie und schlägt. Erstaunt fragen Sie:

$\rightarrow$ Bitte übersetzen Sie den folgenden Satz in Ihren Dialekt und schreiben Sie ihn so auf, wie Sie ihn sagen würden:

Hast du die Uhr flicken lassen?

3. Brunos Holztreppe ist schon wieder kaputt. Was tut er?

$\rightarrow$ Bitte übersetzen Sie den folgenden Satz in Ihren Dialekt und schreiben Sie ihn so auf, wie Sie ihn sagen würden:

Er lässt den Schreiner kommen.

5. Marianne und Peter O. sind bei Mariannes Schwester eingeladen. Nach dem Festessen wollen Marianne und Peter helfen, das Geschirr abzuräumen. Die Schwester winkt jedoch ab und sagt:

$\rightarrow$ Bitte übersetzen Sie den folgenden Satz in Ihren Dialekt und schreiben Sie ihn so auf, wie Sie ihn sagen würden:

Ihr dürft alles liegen lassen.

\section{Anhang 2: Karten}

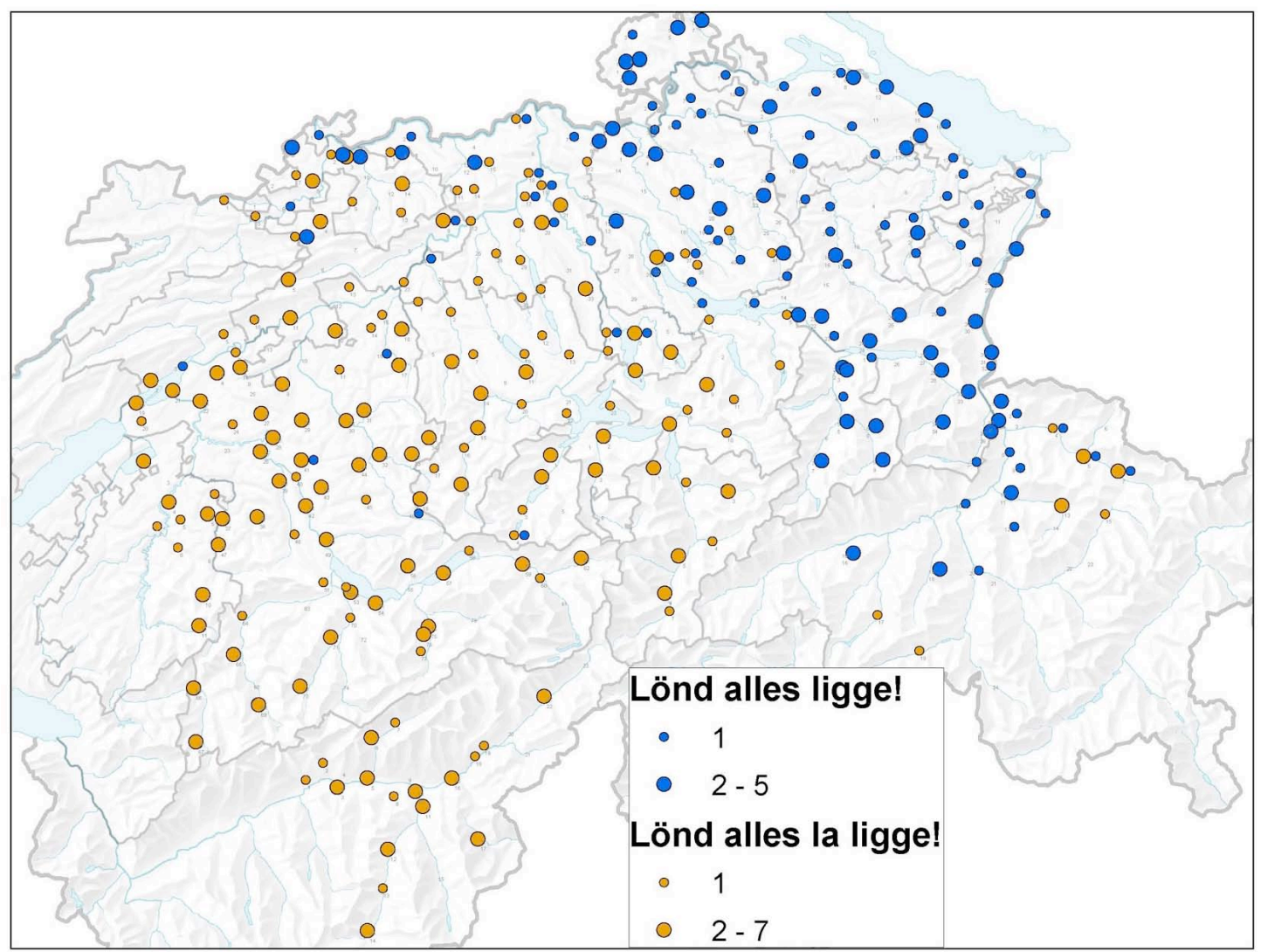

Karte 1: Verteilung der zunehmenden Häufigkeit der Verdoppelung von laa im Imperativ (Lötscher 1993: 196) 


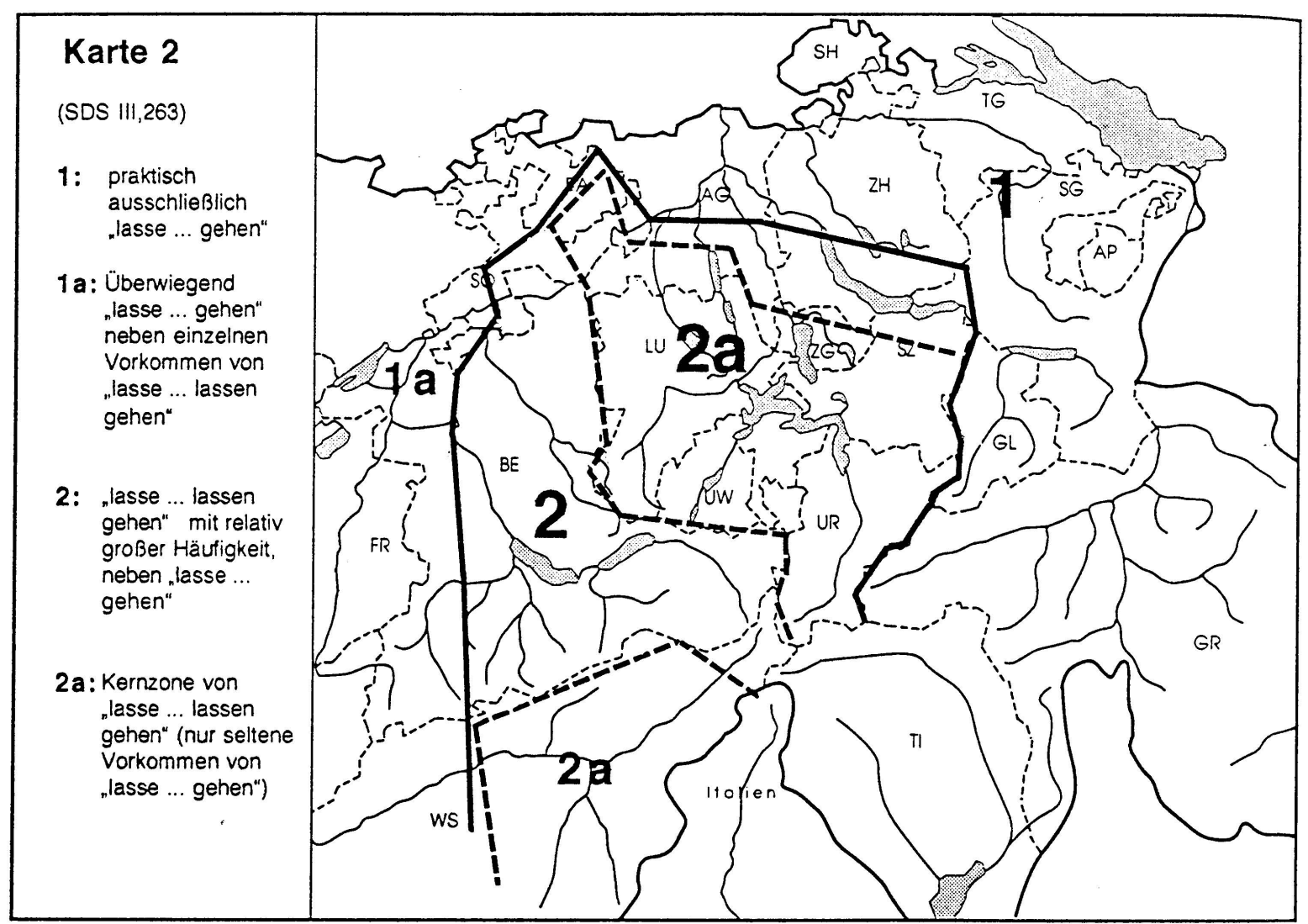

Karte 2: Aufgabe II.5 (SADS) Ihr dürft alles liegen lassen als Imperativ übersetzt (Stand: Dez. 2007) 